\title{
Einige Fragen der Entwicklung der finnisch- ugrischen Trompetenreusen
}

Den Forschern der finnisch-ugrischen Ethnologie ist die Theorie von U. T. Sirelius wohl bekannt, der die verschiedenen, in der öffnung der Sperrvorrichtungen (Wehre) angebrachten Reisigbündel für die althergebrachte Form der Trompetenreusen hält. Die Trompetenreuse ist ein Fischfanggerät ohne Kehle. Die Ausführungen von U. T. Sirelius lauten folgendermassen: "So werden in manchen Gebirgen, wie z.B. in Siebenbürgen (bei den Szeklern), Kaukasien (Lenkoran), im Ural (zwischen Tscheljabinsk und Zlatoust) und an den Gebirgsbächen der oberen Kama (Stein)wehre in Form einer stromabwärts gerichteten Ecke hergestellt, deren in der Spitze angebrachte Öffnung durch ein mit einigen Steinen beschwertes Reisigbündel, in welches der rasche Stromstrich den Fisch hineinwirft und zerschlägt, versperrt wird (Jankó, S. 162, 171, 177). Allgemein üblich ist es wenigstens in Siebenbürgen solche Wehre für die Trampfischerei zu bauen (S. 117), bei deren Ausübung gewöhnlich ein Fischer an der Wehröffnung die Ankunft der Fische erwartet, während zwei andere dieselben von oben herunterscheuchen."

"Ein solches Wehr und eine solche Fangart deuten schon durch ihre Einfachheit in längst entschwundene Zeiten."

"Es ist leicht einzusehen, dass die Fische dabei einerseits durch die scharfen Spitzen des Reisigbündels verletzt wurden und verdarben, und anderseits schwer ans Land $\mathrm{zu}$ bringen waren, da sie die Strömung bald auf den Grund trieb, bald zwischen die Enden der Reiser festklemmte u.s.w. Mit der Zeit musste der Fischer daher auf den Gedanken kommen, dass er durch Verquickung seines eckförmigen Wehres mit 
einer kleineren, aber zugleich transportabeln und mit einem Boden versehenen eckförmigen Vorrichtung die Beute leichter aus dem Wasser emporheben konnte."

"In Frankreich, diesem berühmten Kulturland, hat sich eine solche eckige Vorrichtung (canard) erhalten, wie uns scheint, das Prototyp der Trompetenreuse. Sie ist im Departement du Var neben dem Reisig- und Steinwehr gebräuchlich und wird daselbst aus Schilfrohr, das man mit dünnen Iindenreisern zusammenflicht, gefertigt. Beim Fischen befindet sich im Sterz dieses Gerätes ein Reisigbündel im Wasser, wie es fig. 559 [hier Abb. 1] deutlich veranschaulicht (Daubrée, S. 63). Eine bessere Übergangsform von dem einfachen Bündelfanggerät zu der primitiven Trompetenreuse lässt sich kaum denken.»

"Als eine echte Trompetenreuse könnten wir vielleicht schon das in fig. 560 [hier Abb. 2] abgebildete Fanggerät (canis, canisse) ansprechen, denn sein hinterer Teil, in dem die Fische endgültig verbleiben, ist in der Tat bereits rohrförmig gestaltet, während die Mündungspartie immer noch oben offen ist. Sie begegnet gleichfalls in Frankreich und zwar im Departement Aude (Daubrée, S. 65)."

"Die am weitesten fortgeschrittene französische Form der Trompetenreuse (loun) finden wir in fig. 561 wieder. Sie wird aus Nussbaumholz ca. 2,5 $\mathrm{m}$ lang und an ihrem schmalen Ende, welches mit einem Bündel Schilf verschlossen wird, $20 \mathrm{~cm}$ im Durchmesser gemacht und mit einer Spirale aus Bandholz ausgerüstet. Man erbeutet damit namentlich Aale und stellt das Gerät in die Wehrtore. Heimat Departement Corrèze (Daubrée, S. 210).» ${ }^{1}$

Die oben zitierten Stellen aus dem Buch von U. T. Sirelius zeigen, dass U. T. Sirelius das in die Öffnung der V-förmigen Wehre gelegte Reisigbündel für die althergebrachte Form hält, aus der sich die Entstehung und Entwicklung der Trompetenreuse erklären lässt.

1 U. T. Sirelius, Über die Sperrfischerei bei den finnisch-ugrischen Völkern. Helsinki, 1906. S. 383-384. Mit der Theorie von U. T. Sirelius stimmt auch die Theorie K. Moszyńskis überein. K. Moszy Ńs K I, Kultura ludowa Slowian, Bd. I. Kraków, 1929. S. $75-76$. 


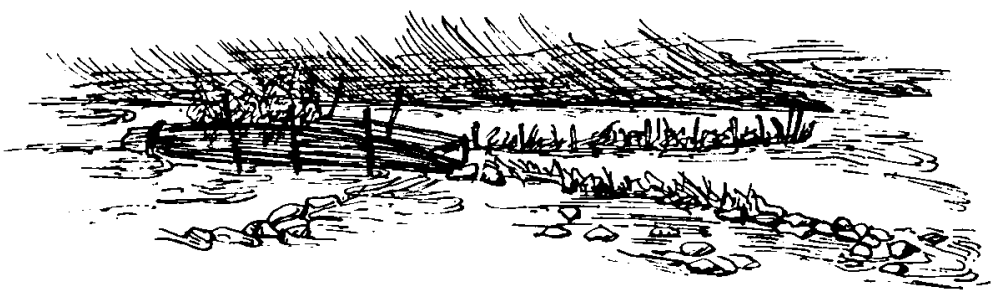

Abb. 1. Sperrvorrichtung zum Fischfang aus Reisig- und Schilfrohrbündel. Frankreich, Depart. Var. Nach M. L. Daubrée-U. T. Sirelius.

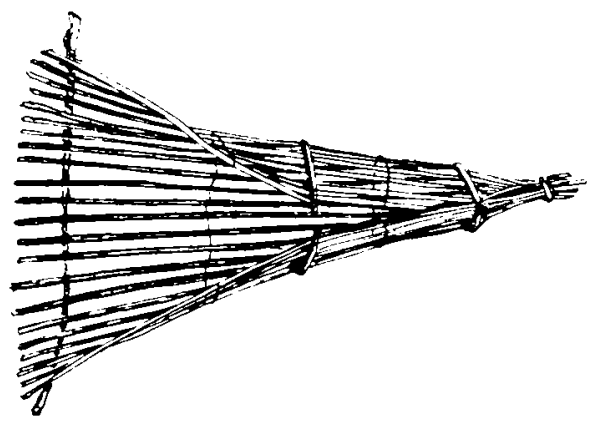

Abb. 2. Kapuzenreuse. Frankreich, Depart.Aude. Nach M. I. Daubrée - U. T. Sirelius.

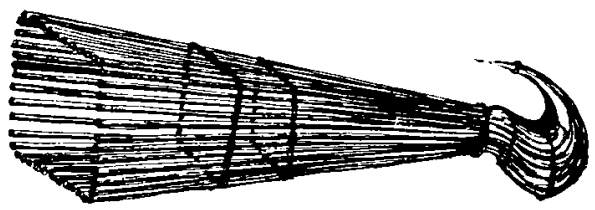

Abb. 3. "Babuschenreuse" der Szekler. Nach O. Herman. 
Die Angaben von U.T. Sirelius über den Fischfang mit Reisigbündel kann ich durch weitere Varianten ergänzen. Diese Varianten lassen sich an den Anfang des Entwicklungsganges stellen, an dessen Ende die Trompetenreusen stehen.

In Siebenbürgen, im breiten Becken des Dragan-Baches und des Kalten- und Warmen-Szamos, die alle eine ziemliche Strömung haben, wird bei den Rumänen so gefischt, dass man Baumzweige, meist Tannenzweige, ins Wasser trägt, woraus ein $1 \mathrm{~m}$ breites Wehr gebaut wird. Auf das aus Zweigen bestehende Wehr werden Steine gelegt, wobei die Zweige auch mit darunter eingeschlagenen Stangen befestigt werden. Das Wasser treibt die Fische unter die Zweige, wobei sich einige Fische auf den spitzen Zweigen aufspiessen. Die Fische nimmt man mit der Hand von den Zweigen ab. Das Wehr heisst im Rumänischen gard 'Zaun, Damm'. Im Siebenbürger Erzgebirge wird der Remete-Bach (bei dem Dorfe Kisgyógypatak) mit Tannen- und Dornrosenzweigen versperrt und die Fische werden aus einer Entfernung von etwa $300 \mathrm{~m}$ bergab oder bergauf getrieben. Das Wasser fliesst durch die Zweige, die Fische aber bleiben an den Zweigen hängen, von wo sie mit der Hand vorsichtig entfernt werden. ${ }^{1}$

G. Inczefi beschreibt in seiner Darstellung der primitiven Fischfangsmethoden der Rumänen aus der Gegend von Naszód (Siebenbürgen) ein Verfahren, bei dem die rumänischen Bauern zahlreiche Tannenzweige im nahen Walde abbrechen und sie zum Bach tragen. Im niedrigeren Bett versperren sie den Bach querdurch. In der Sperrvorrichtung gibt es keine Öffnung. Ein Bauer treibt das Wasser mit einem Ast in der Hand von unten her. Die flüchtenden Fische nehmen die Tannenzweige für ein gutes Versteck und verbergen sich darunter. Wo der Fischer die unter die Zweige gedrängten Fische sieht, fängt er sie mit einer raschen Bewegung. Anstatt der Tannenzweige werden auch die dornigen Äste des Weissdorns (Crataegus oxyacantha) angewandt in der Hoffnung, dass die Fische an den Dornen stecken bleiben. ${ }^{2}$ Diese einfachen

1 Eigene Beobachtungen (1943-1948). Wo die Quellen nicht angegeben sind, teile ich das Material meiner eigenen Feldforschung mit.

2 G. Inczefi, Halászat a Nagy-Szamos felső folyása mentén. (Manuskript). 
Sperrvorrichtungen scheinen bei den Rumänen ziemlich allgemein zu sein, denn auch Gr. Antipa erwähnt in seiner Monographie über die rumänische Fischerei, dass Baumzweige, speziell Tannenzweige von den rumänischen Bauern in den Gebirgsbächen querdurch abgelegt und später die an den Zweigen hängengebliebenen Fische herausgenommen werden. ${ }^{1}$

Merkwürdig sind auch die einfachen Fischfangmethoden, die bei den Ukrainern in den Nordöstlichen Karpaten im Gebrauch sind. Die Huzulen (eine ukrainische ethnische Gruppe) in Máramaros gehen das Wasser entlang und werfen Erlen- und Weidenlaub in den Bach, das mit Steinen heruntergedrückt wird. Dann kehren sie auf demselben Weg zurück, diesmal jedoch schon mit einem Knüppel in der Hand, mit dem sie kräftig auf das Wasser schlagen. Die erschrockenen Fische suchen unter den Erlen- und Weidenblättern Unterschlupf, von wo man sie dann mit der Hand herausnehmen kann. ${ }^{2} \mathrm{~V}$. Vladykov berichtet, dass die ukrainischen Flösser und Waldarbeiter belaubte Baumstämme ins Wasser werfen, die nach einer Zeit herausgehoben werden, woraufhin man die unter den Zweigen zappelnden Fische fängt. ${ }^{3}$ Diese Fischfangmethode habe ich auch selbst bei den Huzulen in der Gegend von Rahó (Kom. Máramaros) beobachtet. Die Rumänen in Nordsiebenbürgen, am Flusse Lápos (Románlápos, Rogoz, Macskamezô) legen junge, kaum 1-2 m hohe Tannen und belaubte Erlenzweige in den Fluss; nach einer Weile hebt man sie schnell heraus, wobei die Fische unter den Zweigen herausgenommen werden. Ein ähnliches Verfahren wird von den ungarischen Bewohnern des Dorfes Domokos am Flusse Lápos angewandt. Hier ist es auch üblich, dass die belaubten Zweige wie Weizengarben zusammengebunden und abends in den Fluss gelegt werden. Am frühen nächsten Morgen nimmt man die Zweiggarben aus dem Wasser und die Fische werden herausgeholt. Ein Bauer legt manchmal 8-10 solcher Astgarben in das Flussbett, die mit Steinen und

1 Gr. Antip A, Pescăria și pescuitul in România. Bucureşti, 1916. S. 90.

2 H. Sztripszky, Ósfoglalkozási dolgok Mármarosból. In: Néprajzi Múzeum Értesítője (= NÉ.), X. Budapest, 1909. S. 219.

3 V. Vladykov, Ryby Podkarpatskoj Rusi i ich glavnejšie sposoby lovli. Ungvár, 1926. S. 90-93. 
Pfählen im Wasser gehalten werden. Neben die Steine wird eine lange Stange gesteckt, an deren oberem Ende ein Halmknoten angebracht wird, was eigentlich als Eigentumsmarke gilt und bedeutet, dass niemand in der Nähe der Stangen fischen, Reusen oder Astgarben auslegen darf.

Es sei hier noch ein Verfahren erwähnt, das gleichfalls bei den Huzulen in Máramaros zum Fangen von Schlammbeissern angewandt wird, wobei man einige mit Steinen beschwerte Schraubstrohbündel vom Ufer ins Wasser herunterlässt, die an im Bachbett befestigte Pfähle gebunden sind. Nachts verstecken sich die Schlammbeisser im Strohbündel. Dann nimmt man die Bündel schnell heraus und die Fische werden aus dem Bündel geschüttelt. ${ }^{1}$

In den Nordkarpaten bauen die Slowaken Steinwehre im Fluss, und die Schmerlen, die sich unter die Steine verziehen, werden mit der Hand herausgenommen. ${ }^{2}$

Bei einer ethnischen Gruppe der Siebenbürger Rumänen, bei den Motzen (im Siebenbürger Erzgebirge) staut man das Wasser durch nebeneinander gelegte Kieselsteine querüber an, so dass der Fisch nicht bergauf schwimmen kann. Viele Forellen finden unter diesen Steinwehren Unterschlupf, und man nimmt sie einzeln mit der Hand heraus. ${ }^{3}$

Die rumänischen Bündel- und Steinwehre sind nicht $V$ förmig, sondern sie verbinden die beiden Ufer in einer geraden Linie. U. T. Sirelius spricht entschieden von V-förmigen Formen, doch geht aus den Angaben von J. Jankó nicht

1 H. Sztripszky, a.a.O. S. 219. - In Nordkurland werden die Neunaugen mit einem ins Wasser hinuntergelassenen, belaubten Erlenastbündel gefangen. (A. Bielenstein, Die Holzbauten und Holzgeräte der Letten, Bd. II. Petrograd, 1918. S. 676). Bei Metz fängt man Krebse mit Reiserbündeln. (M. Borne, Handbuch der Fischzucht und Fischerei. Berlin, 1886. S. 675.) All diese Verfahren stimmen mit dem oben beschriebenen Fischfang der Ungarn, Rumänen und Huzulen überein. (Vgl. noch K. Moszý́ski, a. a. O. S. 25.)

2 R. Bednárik, Slowakische Volkskultur. Pressburg, 1943. S. 195.; R. BEDNÁik, Hmotná kultúra slovenského l'udu. In: Slovenská vlastiveda, Bd. II. Pressburg, 1943. S. 204.

${ }^{3}$ G. Moldován, A románság. Politikai, néprajzi és nyelvészeti közlemények. Nagybesskerek, 1895. Bd. II. S. 17. 
hervor, ob es sich um V-förmige Sperrvorrichtungen oder um jene Wehre handelt, welche die beiden Ufer in gerader Linie verbinden. Eine Sperrvorrichtung aus Latten oder Lattenschirmen, welche die beiden Ufer in gerader Linie verbinden, finden wir bei den Konda-Wogulen. Die Fische sammeln sich an der Wand der Wehre und werden mit Schlaghaken oder mit Hamen herausgeholt. ${ }^{\perp}$ Diese Verzäunung gehört aber schon zu jener Gruppe der Sperrvorrichtungen, die U.T. Sirelius unter der Bezeichnung lältäm darstellt. ${ }^{2}$ Der in den Bach oder Fluss gelegte belaubte Baumstamm, die junge Fichte, der belaubte Ast, das Zweig- und Strohbündel bei den Ukrainern, Rumänen und Ungarn sind primitivere Fischfangsverfahren, als die künstlich gebauten Wehre aus Reisigbündeln und Baumzweigen.

Zwischen den Reisigbündeln, belaubten Baumzweigen, Strohbündeln, Reisigwehren und Trompetenreusen gibt es eine Übergangsform, die U. T. Sirelius - wie oben zitiert aus Frankreich kennt (Abb. 2). Diese Reusenform, die wir Kapuzenreuse nennen können, ist aber nicht nur in Westeuropa, sondern auch in einem grossen Gebiet der Karpaten und der Balkanhalbinsel bekannt. Weder J. Jankó noch U. T. Sirelius haben wahrgenommen, dass $O$. Herman diese Reusenform bei den Szeklern schon vorgefunden und beschrieben hatte. Sie Intersuchten die Beschreibung von 0 . Herman nicht aufmerksam, und hielten die Kapuzenreuse für eine Variante der sog. Bundschuhreuse auf der Abbildung 110 des Buches von 0 . Herman. ${ }^{3}$ Auch $O$. Herman selbst fasste diese Reuse, die seiner Meinung nach "rollkommen pantoffelförmig" sei, als eine Variante der Bundschuh- oder "Babuschenreuse" (Bild 110 in seinem Buche, hier Abb. 3.) auf."

Das ist jedoch ein Irrtum. Die pantoffelförmige Reuse $O$. Hermans aus Siebenbürgen ist eine typische Kapuzenreuse,

1 U. T. Sirelius, a.a.O. S. 18.

¿ L. T. Sirelics, a.a.O. S. $354-355$.

3 J. Jaxkó, Herkunft der magyarischen Fischerei. Budapest-Lipcse, 1900. S. 178-179; C. T. Sirelits, a.a.O. S. 114 .

4 O. Herman, A magyar halászat könyve. Bd. I. Budapest, 1887. S. $231-232$. 
über die wir mehrere ausführliche Beschreibungen besitzen. Die Ungarn im Tale des Lápos (Siebenbürgen) kennen ein Fischfanggerät mit der Bezeichnung szelep (Abb. 4.), das eigentlich ein dreieckiges Rutengeflecht ist. Es besteht aus einem ungefähr $190 \mathrm{~cm}$ langen, $70 \mathrm{~cm}$ breiten, aufwärts immer engeren Rutengeflecht, das in einer flachen Kapuze oder in einem Korb endet. Der Fluss Lápos und die kleineren Bäche werden mit Steinen keilförmig angestaut und der szelep wird in die Öffnung des Wehrs (gerdus) gegen den Wasserlauf gelegt. Die schnelle Strömung des Wassers reisst die Fische in den engen - nur locker zugedeckten - Korb, in die Kapuze hinein, wo sie stecken bleiben. Die korb- bzw. kapuzenförmige Spitze des szelep wird oft mit Baumzweigen bedeckt, man steckt Zweige darauf, damit die hineingerissenen Fische nicht freikommen können. Mit dem szelep kann man nur in niedrigen Gewässern mit schneller Strömung Fische fangen. ${ }^{1}$ Dieselbe Kapuzenreuse ist bei den Szeklern in Marostorda unter der Benennung lésza oder lészka bekannt." Als eine Variante der Kapuzenreuse gilt die aus Weidenruten geflochtene, löffelförmige Reuse, die den Ipoly entlang (Dorf Bernece) von den Ungarn angewandt wird (Abb. 5.). ${ }^{3}$

Die bei den Szeklern und im Tale des Lápos benutzte Kapuzenreuse ist auch bei den Rumänen bekannt. Sie wird von den Rumänen, die im Gebiete des Kalten-Szamos mit schneller Strömung wohnen, unter Steinen abgelegt. Manchmal baut man auch ein V-förmiges Steinwehr dazu, in dessen Öffnung die Reuse gestellt wird. Die Reuse wird im Rumänischen leasă genannt (Abb. 6.) Diese Reuse wird auch von den Rumänen im nördlichen Teil Siebenbürgens (im Tale der Iza, rum. leasă; Dragomérfalva, rum. vârsoc) verwendet. Manchmal wird sie in die Öffnung eines keilförmigen Steinwehres gelegt. ${ }^{*}$ Die Kapuzenreuse entging selbst der Auf-

1 B. Gunda, Néprajzi gyüjtőuton. Debrecen, 1956. S. 47.

2 H. Sztripszky, Adatok Erdély őshalászatához. In: NE. III. 1902. S. 178 .

3 I. Györffy, Az ipolymenti halászat. In: NE. XXV. 1933. S. 25.

4 M. Roska, Adatok az Iza halászatához. In: Közlemények az Erdélyi Nemzeti Múzeum Érem- és Régiségtárából, Bd. III. Kolozsvár, 1943. S. $190,192$. 


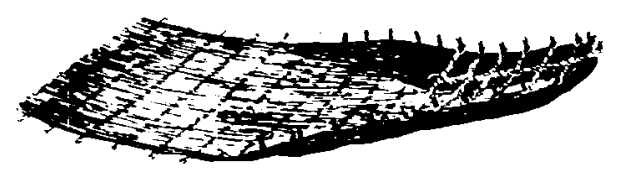

Abb. 4. Kapuzenreuse der Lngarn im Lápos-Tal. Domokos, Lápos-Tal. Xord-Siebenbürgen. Rumänien.

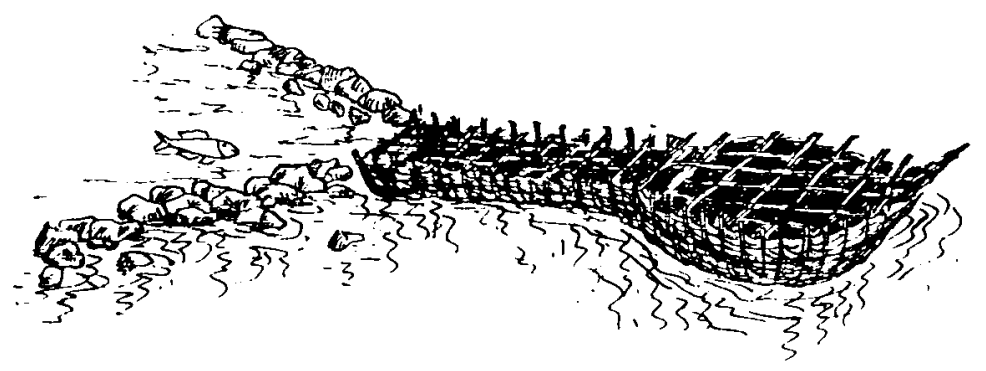

Abl. 5. Lngarische Kapuzenreuse. Bernece an Flusse Ipoly, Ingartn. Vach I. Györffy.

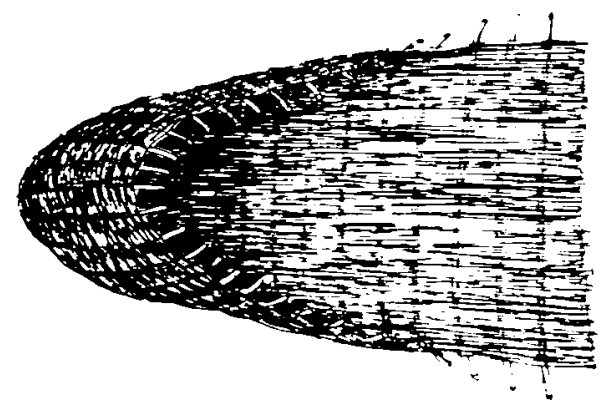

Abb. 6. Rumänische Kapuzenreuse. Dorf Hideg-Szamos, Gyaluer Schneeberge, Siebenbürgen, Rumänien. 
merksamkeit voll (Xl: Antipa nicht. Seiner Beschreibung nach werde dieses Fischfanggerät in Crebirgsbächen von den Rumänen angewandt; der Name dafür laute im Rumänischen leasă. Die Reusen sind nicht gross (40-50 cm lang), ihr Ende steigt etwas an, ihr breiter werdender 'Teil wird mit Steinen am Boden des Baches gehalten. Gr. Antipa gibt auch eine Darstellung der Kapuzenreuse von einem Bach der Bistritz (Abb. 7.). Im Banat (im südwestlichen Teile Siebenbürgens) wird eine Art von Reuse aus Schilfrohr von den Rumänen verfertigt, deren Ende die Form einer Kapuze hat. Man legt belaubte Zweige darauf, welche die Fische daran hindern, sich aus der Reuse zu schnellen. Zur Aufstellung der Reuse werden auch Steinwehre gebaut. In den Südkarpaten (Oltenia) wird diese rumänische Reuse (legănus, povărasc) aus zwei zusammengenähten Lindenbaststücken hergestellt (Abb. 8.), die etwa $3 \mathrm{~m}$ lang sind. Sie wird in der Gegend von Baia de Aramă am Bache Motru benutzt. Ihre Leitwand (das Wehr) besteht aus Rutengeflecht. ${ }^{1}$ Die Anwendung der Kapuzenreuse bei den Ukrainern in Máramaros wird von H. Sztripszky ausführlich dargelegt. In der Gestalt entspricht diese Kapuzenreuse völlig jener Form, die aus dem Tale des Lápos bekannt ist. Ihre Länge ist oft $3 \mathrm{~m}$, unten ist sie $1,50 \mathrm{~m}$ breit. Von der Mitte gekrümmt biegt sie sich etwas aufwärts, weil so der Zug des Wassers stärker ist. Die grösseren Formen (soup) werden in Flüssen, die kleineren (páljisok) in Bächen gebraucht. Das zu der Reuse gebaute Steinwehr (lijz) hat VForm. ${ }^{2}$ Die Benûtzung dieser Reuse wird in mehreren Beschreibungen von den ukrainischen Volksgruppen (Huzulen) in der Karpato-Ukraine, in der Bukowina und in Galizien erwähnt, ${ }^{3}$ V. Vladykov behandelt sie besonders eingehend.

1 Gr. Antipa, a.a.O. S. $101-102$.

2 H. Sztripszky, Ôsfoglalkozási dolgok ... S. 220.

3 W. Szuchiewicz, Huculszczyzna, Bd. I. Kraków, 1902. S. 2j1; R. Fr. KaINDL, Beiträge zur Ethnologie und Volkskunde der Huzulen. Globus, LXIX. Braunschweig, 1896. S. 92.; R. Fr. KAINDL, Die Fischerei bei den Huzulen. Oesterreichische Forst- und Jagdzeitung, Jhg. 16. Wien, 1898. S. 144.; K. Moszý́sk , Kultura ludowa Stowian, Bd. I. Kraków, 1929. S. 76-77. 


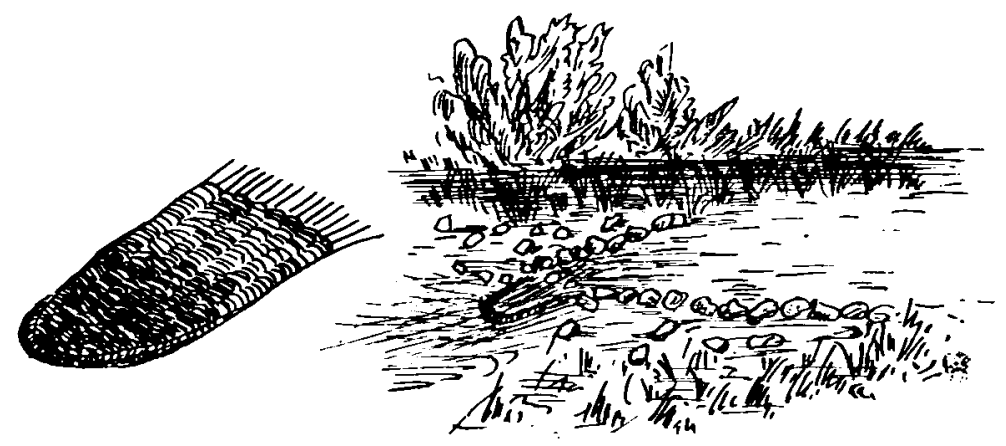

Abh. 7. Rumänische Kapuzenreuse. Tal des Flusses Beszterce (Bistritz), Rumänien. Nach Gr. Antipa.

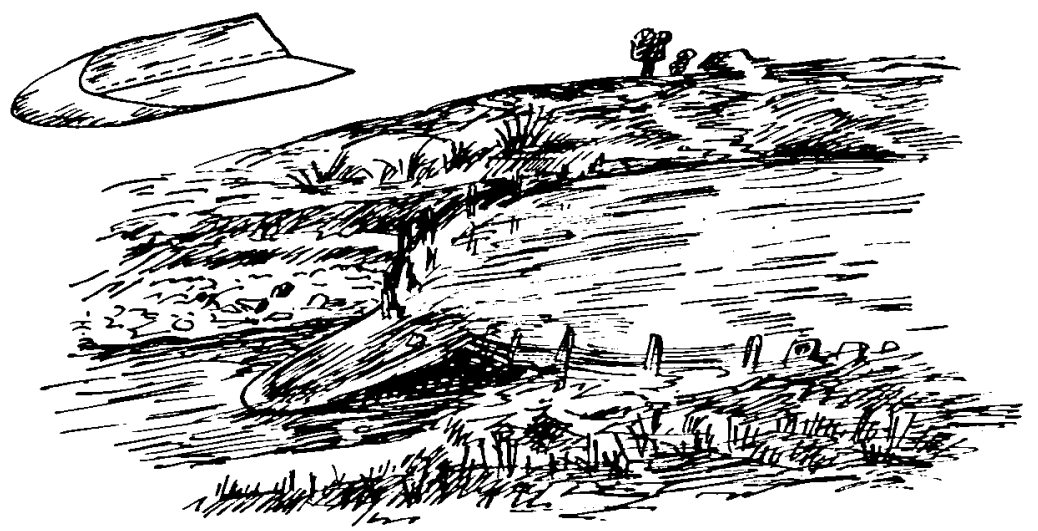

Abb. 8. Rumänische Kapuzenreuse aus Lindenbast. Gegend von Baia de Aramă am Bache Motru, Südkarpaten, Rumänien. Nach Gr. Antipa. 
Seinen Untersuchungen zufolge ist diese primitive Reusenform sehr verbreitet und hat ein crrösseres Format (palěsok, bzw. soup). Dementsprechend ist sie $1-2,50 \mathrm{~m}$ lang und ragt vorne etwas empor. Sie dient dem Fischfang in Gewässern mit schnellem Lauf, so dass sie in der Öffnung von V-förmigen Ruten- oder Steinwehren angebracht ist. Das offenc, in einem Körbchen (koš) mündende Ende der Kapuzenreuse wird mit Steinen und mit einem Stock unterstützt, damit sie aus dem Wasser ilufragt. Es ist auch üblich, dass man einen Sack auf das Ende der Kapuzenrense zieht. Die Flügel der Reuse, die sich in Form eines Fächers ausbreiten, werden mit Steinen befestigt. Ias Fischfanggerät wird nachts gebraucht, tagsüber wird es versteckt, sein Platz ist jedoch an dem Stein- oder Rutenwehr zu erkennen. Mit dieser primitiven Reuse wird nach dem Abzug des Hochwassers im Frühling bis zum Erscheinen des Hochwassers im Herbst gefischt. Es kommt vor, dass eine etwa $1 \mathrm{~m}$ hohe und 15-20 $\mathrm{m}$ lange Flechtwand der Breite des Baches oder des Flusses entsprechend im Wasser gezogen wird, wobei man die Fische in die Kapuzenreuse treibt. Die Flechtwand wird ron mehreren Leuten in senkrechter Stellung zum Grund des Bettes gehalten, und die beiden Enden werden von je einem Fischer gezogen. I)as Treiben der Fische mit der Flechtwand beginnt aus einer Entfernung ron einem halben oder einem Kilometer vor der Kapuzenreuse. ${ }^{1}$

Die Kapuzenreuse ist auch bei den Slowaken (Kom. Trencsén, leska im Slowakischen) bekannt. ${ }^{2}$ Sie wird auch von den Goralen (eine polnische ethnische Gruppe in der Hohen Tatra) verwendet und trägt die Bezeichnung 'Bundschuh' (kierpec). ${ }^{3}$ Aus der Angabe ron K. Moszyński geht hervor, dass der Gebrauch der kapuzenförmigen Reuse in den Nordkarpaten ziemlich allgemein war. ${ }^{4}$

Im wesentlichen ist das Fischfanggerät der Bulgaren diesem Gerät ähnlich; es ist eigentlich nur ein dünnes, in Richtung

1 V. VLADYKov, a.a.O. S. $94-103$.

2 R. BEDNARIK, a.a.O. S. 196, bzw. S. 208.

3 K. Moszýśsi, a.a.O. S. 77.

4 K. Moszý́ski, a.a.O. S. $76-77$. 
des Wasserlaufs aufgestelltes Rutengeflecht, das vorne mit Steinen unterstützt wird; die schnelle Wasserströmung schleudert die Fische in das Geflecht hinein. ${ }^{1}$ Kapuzenförmige Reusen sind auch aus den Beschreibungen von den Serben bekannt. $^{2} \mathrm{~V}$. Curčić berichtet über einen aus Ruten geflochtenen Korb, der die Form eines offenen Kastens hat und unter der Rinne der Wassermühle angebracht wird; die Fische werden durch die schnelle Strömung in den Korb hineingerissen, wobei sie manchmal fast zerschmettert werden. ${ }^{3}$ Dieses Gerät mag als eine spezielle Variante der Kapuzenreuse gelten.

Mit den ungarischen, rumänischen, ukrainischen, slowakischen, goralischen usw. Reusen ist jene Reuse identisch, die in dem Buch von U. T. Sirelius aus Frankreich dargestellt ist, ${ }^{4}$ deren Identität mit den Kapuzenreusen der Huzulen schon von $A$. Haberlandt entdeckt wurde. ${ }^{5}$ Dieser Reusentyp ist von $\mathrm{K}$. Moszyński auch aus Portugal belegt. ${ }^{6}$

Die Kapuzenreuse gilt zweifellos als althergebrachte und charakteristische Reusenform der Karpaten und des BalkanGebiets. Aus den westeuropäischen Analogien lässt sich schliessen, dass die Kapuzenreuse eine alteuropäische Reusenform darstellt, die sowohl in Westeuropa wie auch in dem Gebiet der Karpaten und auf der Balkanhalbinsel erhalten ist.

Aber nicht nur die Kapuzenreuse, sondern auch eine weiter entwickelte Variante derselben - die Trompetenreuse - ist im Kulturraum der Karpaten und der Balkanhalbinsel bekannt.

1 K. Moszý́ski, a.a.O. S. 76; Chr. VAKarelski, Bit' na trakijskitè i maloazijskitè b'lgari. Trakijski Sbornik', Bd. V. Sofia, 1935. S. 17.

2 K. Moszý́skı, a.a.O. S. 76; B. Drobnjaković, Ribolov na Drini. Beograd, 1934. S. 29.

3 V. Ćunčı́, Die volkstümliche Fischerei in Bosnien und der Herzegowina mit besonderer Berücksichtigung der Savefischerei bei Dônja Dolina. Wissenschaftliche Mitteilungen aus Bosnien und der Herzegowina, Bd. XII. Wien, 1912. S. 544-545.

4 U. T. Sirelius, a.a.O. S. $383-384$.

5 A. Haberlandt, Die volkstümliche Kultur Europas in ihrer geschichtlichen Entwicklung. In: G. Buschas, Illustrierte Völkerkunde, Bd. II. Wweiter Teil. Stuttgart, 1926. S. 324.

6 K. Moszý́ski, a.a.O. S. 76. 
Die Trompetenreuse ohne Kehle wird von den Ungarn (in Bodrogköz) mit dem Namen haboknya bezeichnet. Sie ist eigentlich ein $2-3 \mathrm{~m}$ langes, aus Ruten geflochtenes, walzenförmiges Rohr, das sich an der Mündung plötzlich erweitert (Abb. 9.). Sie wird in den Kanälen des Überschwemmungsgebietes der Theiss zur Zeit des Fischfangs im Frühjahr ausgelegt. ${ }^{1}$ Eine charakteristische Trompetenreuse wird im alten Komitat Bereg gebraucht; sie heisst dort hurca. Sie ist $130 \mathrm{~cm}$ lang und hat an der Mündung einen Durchmesser von $55 \mathrm{~cm}$ (Abb. 10.). Ein V-förmiges Wehr wird aus Stein und Ruten gebaut, in dessen Öffnung dann die Reuse gestellt wird. Die Bäche Román und Borsova, die eine schnelle Strömung haben, bringen die Fische in diese hurca. ${ }^{2}$ Am Oberlauf der Theiss wird die Trompetenreuse gleichfalls von den Ungarn gebraucht; hier wird damit im Schlamm der sumpfigen Bäche gefischt. Diese Trompetenreuse ist etwa $2 \mathrm{~m}$ lang und ihr Rutengeflecht ist für kleine Fische dichter, für grössere Fische dünner geflochten (Abb. 11.). ${ }^{3}$

Mehrere westliche (französische, englische) und östliche (russische, sibirische, altaische, tatarische) Varianten der Trompetenreuse werden von U. T. Sirelius dargestellt. Die Trompetenreuse ist auch bei den verschiedenen finnischugrischen Völkern (bei den Finnen, Esten, Syrjänen, Ostjaken und Wogulen) vorhanden und U.T. Sirelius sagt folgendes über ihre Entstehung: "Die Trompetenreusen haben bereits in finnisch-ugrischer Zeit in Gebrauch sein können". "Von einigen Lokalformen abgesehen waren "die finnischen Trompetenreusen schon in Nordwestrussland bekannt.... ${ }^{5}$ U. T. Sirelius sieht die ungarische "Babuschenreuse», die im Buch von O. Herman dargestellt ist (Abb. 3.), als eine Lokalform an, demgegenüber ist J. Jankó der Ansicht, dass sie ugrischer

1 I. Ecsedi, Népies halászat a Közép-Tiszán és a tiszántúli kisvizeken. Debrecen, 1934. S. 35.

2 Eigene Beobachtungen.

3 J. Babus, A lónyai vizek néprajza. In: Néprajzi Közlemények, Bd. IV. Nr. 3. Budapest, 1959. S. 53.

4 U. T. Sirelius, a.a.O. S. 463.

5 U. T. Sirelius, a.a.O. S. 463. 


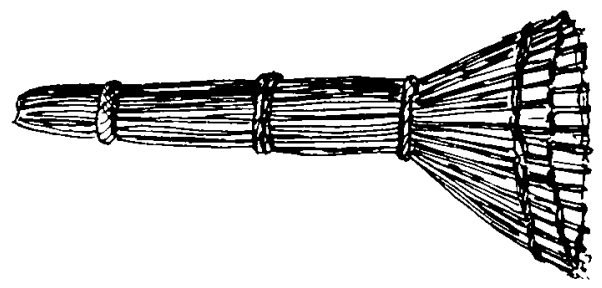

Abb. 9. Ungarische Trompetenreuse. Bodrogköz, Lngarn. Aach I. Ecsedi•

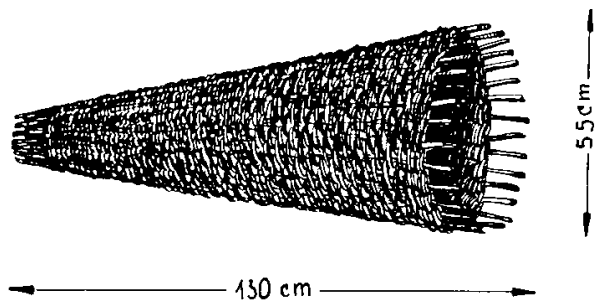

Abb. 10. Ungarische Trompetenreuse. Beregújfalu, Karpato-Ukraine.

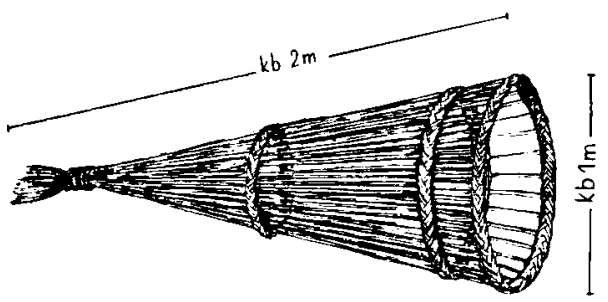

1bb. 11. Ungarische Trompetenreuse. Bátyu, Gegend an der oberen Theiss, Karpato-Ukraine. Nach J. Babus. 
Herkunft sei. ${ }^{1}$ J. Jankó begründet seine Auffassung mit der Feststellung, dass Reusen ohne Kehle nur von den OstjakenWogulen und von den Ungarn bekannt sind. ${ }^{2}$

Was die im Buche von O. Herman dargestellte ungarische (szeklerische) Babuschenreuse (Abb. 3.) betrifft, hat U.T. Sirelius zweifellos recht. Diese Babuschenreuse hat man seit dem Erscheinen des Buches von O. Herman in ungarischem Kulturgebiet nicht vorgefunden. Durch eine erneute Erforschung der Fischerei in Siebenbürgen sollte man feststellen, ob diese Form wirklich in Gebrauch war. Die Reuse von $O$. Herman kann man hinsichtlich ihrer Form nicht mit den eigentlichen Trompetenreusen vergleichen, und U. T. Sirelius hält sie mit Recht für eine Lokalform. Typische Formen der Trompetenreusen wurden aber seit dem Erscheinen der Werke von O. Herman, J. Jankó und U. T. Sirelius - wie wir oben gesehen haben - von mehreren Verfassern im ungarischen Kulturgebiet dargestellt (Abb. 9-11.). Leider ist die Etymologie der Bezeichnungen (hurca, haboknya) nicht geklärt. Wenn die finnischen Trompetenreusen bereits in finnisch-ugrischer Zeit bekannt sein konnten, so ist es nicht ausgeschlossen, dass auch die ungarischen Trompetenreusen aus derselben Zeit herrühren. Die Frage wird dadurch kompliziert, dass wir seit den Forschungen von U. T. Sirelius Kenntnis vom Vorkommen typischer Trompetenreusen in Polen (in Polesie, im kurpischen Gebiet, im nordöstlichen Teile des ehemaligen polnischen Weissrusslands) haben. ${ }^{3}$ Solche Reusen sind auch am Flusse Save in Bosnien in Gebrauch. ${ }^{4}$ Selbst nördlich vom polnischen Gebiet, bei den Letten, kommen solche Trompetenreusen vor. ${ }^{5}$

1 L. T. Sirelius, a.a.O. S. 463; J. JANkó, a.a.O. S. 182, 199.

2 J. JANKó, a.a.O. S. 182.

3 T. Seweryn, Wiersza lejowata. Lud Slowiański, Bd. II. Kraków, 1931. B S. 214-216; J. OLEdzk I, Rybołówstwo na terenie kurpiowskiej puszczy zielonej od konca XIX do polówy XX. wieku. In: A. Kutrze baPosnarowa, Kurpie puszcza zielona. Wrocław-Warszawa-Kraków, 1962. S. $181-182$. K. Moszy Ński, a.a.O. S. 77.

4 V. Curčić, a.a.O. S. $537-539$.

5 A. Bielenstein, Die Holzbauten und Holzgeräte der Letten, Bd. II. Petrograd, 1918. S. 673.; Z. Ligers, Die Volkskultur der Letten, Bd. I. Riga, 1942. S. 54.; Z. LIgers, Ethnographie Lettone, Bd. I. 
Bei der Erforschung der ungarischen Trompetenreusen sollte man diese Tatsache nicht ausser acht lassen. Merkwürdig ist, dass I. Manninen über den Ursprung der Trompetenreuse in Estland nichts Näheres sagt. Er beruft sich nur auf U.T. Sirelius, dass sie "die allerälteste primitivste Reusenform» sei. ${ }^{1}$

Als Zusammenfassung der obigen Ausführungen können wir folgendes feststellen:

1. Die Reisig- und Halmbündel, die belaubten Zweige, die Sperrvorrichtungen aus Reisig und Zweigen gelten als primitive Fischfanggeräte der Karpaten und können die erste Stelle in jenem Entwicklungsverlauf einnehmen, der zur Ausgestaltung der Trompetenreusen führt. Die von mir hier mitgeteilten Fischfangmethoden sind viel primitiver als die bezüglichen Angaben von U. T. Sirelius und J. Jankó.

2. Die Kapuzenreusen sind im Gebiet der Karpaten und auf der Balkanhalbinsel (bei den Ungarn, Rumänen, Ukrainern, Slowaken, Goralen, Serben usw.) vorzufinden, sie kommen jedoch auch in Westeuropa (Portugal, Frankreich) vor und können als alteuropäische Fischfanggeräte angesehen werden.

3. Die typischen Trompetenreusen sind auch bei den Ungarn bekannt. Sie mögen aus der finnisch-ugrischen Zeit herrühren, können aber auch Fischfanggeräte mitteleuropäisch-karpatischen Ursprungs sein, da Analogien bei den Letten, Polen und im nördlichen Teil der Balkanhalbinsel nachzuweisen sind. Ihr lokaler Ursprung wird durch die Tatsache bestätigt, dass die Vorformen der Trompetenreusen (Kapuzenreuse, Reisig-

Bâle, 1954. S. 101; S. Cimmermanis, Soldīdenu zveja Lejasciemā un Belava. In: Arheologija un etnografija, Bd. IV. Riga, 1962. S. 175. Die Trompetenreusen kommen auch bei den Grusinern im Kaukasus vor (Sammlung des Ethnographischen Museums der Völker der Sowjetunion, Leningrad). Die Reusen ohne Kehle waren scheinbar auch an der Mur in Österreich bekannt (vgl. J. WALLER, Beiträge zur Geschichte des Fischereiwesens in der Steiermark. Archiv für Fischereigeschichte. Heft 9. Berlin, 1917. S. 34.).

1 I. Manninen, Die Sachkultur Estlands, Bd. I. Tartu, 1931. S. 231. Vgl. noch I. Manninen, Kalastus. In: Suomen Suku, Bd. III. Helsinki, 1934. S. $67-68$. 
und Halmbündel als Fischfanggeräte) auch im Gebiete der Karpaten sehr häufig vorkommen.

4. Die Forschungsergebnisse von J. Jankó und U. T. Sirelius über die Fischerei der finnisch-ugrischen Völker bedürfen einer Überprüfung.

Es sei noch erwähnt, dass die Theorie von J. Jankó und U. T. Sirelius auch von B. Bonnerjea übernommen wurde, der eine sehr anregende Zusammenfassung der Fischerei der finnisch-ugrischen Völker, auch der Ungarn gab. ${ }^{1}$ Diese Zusammenfassung entging merkwürdigerweise der Aufmerksamkeit der Forscher der ungarischen Fischerei, wie auch I. Györffy keine Kenntnis von den Ergebnissen von U. T. Sirelius nahm, als er im Werk $A$ magyarság néprajza (Ethnographie des Ungarntums, Bd. II. Budapest, $1942 .^{2}$ S. 46-82.) über die ungarische Fischerei schrieb. Das andere Problem, dem vom Gesichtspunkt unserer Untersuchungen eine besondere Aufmerksamkeit gewidmet werden soll, ist enthalten in der vielseitigen Theorie I. V. Vasil'evs, der die Entwicklungsgeschichte der Sperrfischerei der Ob-Ugrier untersucht und einige Theorien von U. T. Sirelius widerlegt. Seiner Schilderung nach verwenden die Wogulen an der Konda zur Zeit der Überflutungen im Herbst und Frühjahr eine Sperrvorrichtung, bei der dem Fischfang die Tatsache zugrunde liegt, dass sich der Fisch, während er nach einem Durchgang sucht, unter dem Reisig versteckt, wobei er sich verirrt. ${ }^{2}$ Diese Sperrvorrichtung stimmt mit den oben dargestellten Reisigwehren überein. I. V. Vasil'ev bringt die Trompetenreusen nicht mit derartigen einfachen Sperrvorrichtungen in Zusammenhang. Die mit einer Öffnung versehenen Wehre aus Reisig, Zweigen usw. weisen gemeinsame Züge mit der Treibjagd durch keilförmige Verzäunungen auf, die bei den Rentierjägern in Sibirien üblich ist. Im Wortschatz sind auch etymologische

1 B. Bonnerjea, La pêche chez les peuples finno-ougriens. L'Anthropologie, tom. 49. Paris, 1939-1940. S. 684, 689-690.

2 V. I. VAsil'ev, Problema proischoždenia orudij zapornogo ribolovstva obskich ugrov. Sibirskij etnograficeskij sbornik, IV. Trudy Instituta Etnografii im. N.N. Miklucho-Maklaja, Nov. Ser. Tom. LXXVIII. Moskva, 1962. S. 139. 
Beweise der Beziehungen zwischen solcher Jagd und Fischerei vorhanden (im Wogulischen ula 'Jagdzaun', ülat 'Sperrvorrichtung der Fischer zur Aufstellung von važan'). ${ }^{1}$ Aus solchen Sperrvorrichtungen des Fischfangs entwickelten sich die Fischzäune, die zur Zeit der finnisch-ugrischen Gemeinschaft (im IV.-III. Jahrtausend v. Chr.) bekannt waren. Das hohe Alter der Fischzäune wird durch die Reste von Fischzäunen aus der neolithischen Zeit bestätigt, die im Mündungsgebiet der Oka und anderwärts vorgefunden wurden. ${ }^{2}$ Nach der Meinung von I. V. Vasil'ev sollen die Trompetenreusen zur Zeit der finnisch-ugrischen Gemeinschaft nicht gebräuchlich gewesen sein, wie von U. T. Sirelius behauptet wird, obwohl die Reste einer Trompetenreuse (rukav) mit Steinwehr, die in einer Tiefe von $2 \mathrm{~m}$ (der Fund wurde nicht datiert) im westlichen Uralgebirge, in der Nähe von Jaswa, aufgefunden wurden, für eine sehr alte Verwendung dieses Fischfanggeräts zeugen. Vom Gesichtspunkt des finnisch-ugrischen Ursprungs kann der etymologische Vergleich B. Collinders nach Ansicht des sowjetischen Ethnographen nicht ausschlaggebend sein: tscher. pos fish-trap (made of rods) / wog. poos fish-trap / ostjak. pas, pos long, narrow fish-trap or fyke, vgl. B. Collinder, Fenno-Ugric Vocabulary, Stockholm, 1955. S. 109. Nach der Auffassung von V. I. Vasil'ev erfolgte die Entstehung der Trompetenreuse in einem späteren Zeitabschnitt der Auflösung der finnisch-ugrischen Gemeinschaft (zweite Hälfte des III. Jahrtausends v. Chr.). Zur Unterstützung dieser Annahme vermag er - wie er auch selbst zugesteht - keine genügend überzeugenden Beweise zu liefern; als Argument gilt ihm lediglich, dass eine etymologisch gemeinsame Bezeichnung dieses Fischfanggeräts in den finnisch-ugrischen Sprachen ganz und gar fehlt. ${ }^{3}$

\footnotetext{
1 I. V. VASIL'EV, a.a.O. S. 141.

2 I. V. VASIL'EV, a.a.O. S. 142-143.

3 I. V. VAsil'ev, a.a.O. S. 144-145. - V. I. Vasil'ev ist der Ansicht, dass die Figuren der von N. V. Cernecov publizierten, verzierten Keramik (Anfang des II. Jahrtausends v. Chr. West-Sibirien, im Norden von Swerdlowsk: am Flusse Rež, Ajatskoe-See) Reusen mit Kehle darstellen. Nach N. V. Cernecov sind die dargestellten Figuren als »mit Fallen ver-
} 
sehene Zäune, Fangvorrichtungen der Jäger und Fischer aufzufassen». (I. V. Vasil'ev a.a.O. S. 146; N. V. Cernecov, Nyugat-szibériai sziklarajzok. In: Ethnographia, LXX. Budapest, 1959. S. 8-10.) Es ist fraglich, ob diese Bilder überhaupt irgendeine Jagdfalle oder ein Fischfanggerät darstellen sollen.

Béla Gunda 